\title{
MASSIVE STAR FORMATION: OBSERVATIONAL CONSTRAINTS
}

\author{
ED CHURCHWELL \\ Dept. of Astronomy, University of Wisconsin \\ 475 N. Charter St.,Madison, WI, 53706, USA
}

\section{Introduction}

Observations during the past several years strongly imply that virtually every star, independent of final mass, goes through a phase of rapid outflow simultaneously with rapid accretion during formation. The structure and properties of outflows and accretion disks associated with low-mass star formation has received intensive observational attention during the past several years (see the reviews and references in Lada 1985; Edwards, Ray, and Mundt 1993; Fukui et al. 1993; and this symposium). Young stellar objects (YSOs) with $\mathrm{L}_{b o l}<10^{3} \mathrm{~L}_{\odot}$ will be referred to as "low-mass" stars in this review. The range of physical properties of $\mathrm{CO}$ outflows associated with YSOs of all masses are enormous, see Fukui et al. (1993). I will focus attention in this review on what we know about massive YSOs and their environments.

\section{Stellar Luminosity and Outflow Properties}

Edwards, Ray, and Mundt (1993), Cabrit and Bertout (1992, hereafter CB92), and others have shown that the bolometric luminosity $\left(\mathrm{L}_{b o l}\right)$ of the central star is correlated with various outflow properties such as the outflow mechanical luminosity $\left(\mathrm{L}_{f}\right)$, the momentum flux $\left(\dot{P}_{f}\right)$ or equivalently the driving force $\left(\mathrm{F}_{f}\right)$, and the mass outflow rate $\left(\dot{M}_{f}\right)$. Clearly, the outflow properties are not independent of the luminosity (or the mass) of the central star. However, the mechanism responsible for driving and collimating the outflows remains unresolved. CB92 suggested that the flows may be driven by an ionized stellar wind based on the fairly tight correlation of the intrinsic $6 \mathrm{~cm}$ continuum flux density $\left(\left(\mathrm{S}_{6} / \mathrm{d}^{2}\right) \propto \mathrm{N}_{c}\right)$ with $\mathrm{L}_{f}$ and $\dot{P}_{f}$. Other suggestions have also been proposed including several versions of accretion 


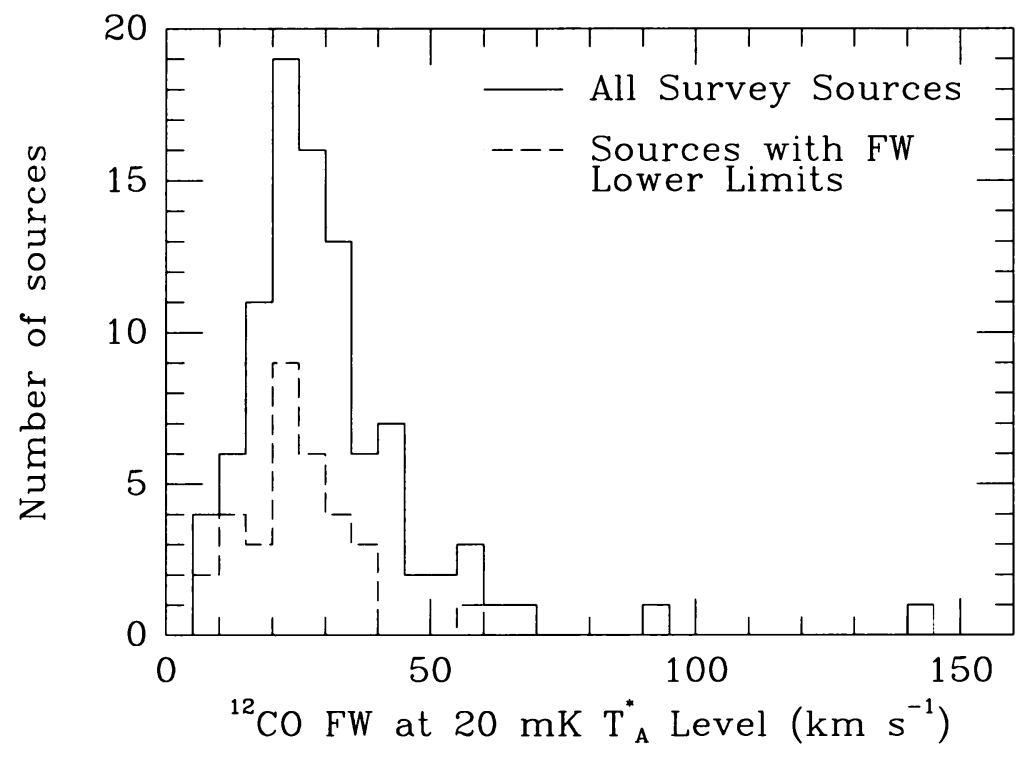

Figure 1. Distribution of measured ${ }^{12} \mathrm{CO}(1-0)$ line full-widths at $20 \mathrm{mK}$ from SC95.

driven outflows involving magnetic fields and rotation, photoevaporation of accretion disks coupled with radiative acceleration, etc. I will return to this issue later in regard to massive star outflows.

\section{The Frequency of Massive Star Outflows}

Motivated by the fact that no systematic study of the frequency of outflows associated with massive stars existed, Shepherd and Churchwell (1995; hereafter SC95) undertook a CO (1-0) line profile survey of 94 massive star formation regions using the NRAO $12 \mathrm{~m}$ telescope (HPBW 60 ") to identify potential outflow sources. They found fully $90 \%$ of their sample was associated with high velocity (HV) gas. At the $1 \sigma \mathrm{T}_{A}^{*}$ level, only $10 \%$ had a full width FW $<15 \mathrm{~km} \mathrm{~s}^{-1} ; 49 \%$ had $15<\mathrm{FW}<30 \mathrm{~km} \mathrm{~s}^{-1} ; 30 \%$ had $30<\mathrm{FW}<45$ $\mathrm{km} \mathrm{s}^{-1}$; and $11 \%$ had $\mathrm{FW}>45 \mathrm{~km} \mathrm{~s}^{-1}$. The distribution of $\mathrm{FWs}$ at $20 \mathrm{mK}$ is shown in Fig. 1 which shows that most ${ }^{12} \mathrm{CO}(1-0)$ FWs lie in the range $20-30 \mathrm{~km} \mathrm{~s}^{-1}$ toward massive star formation regions when observed with a spatial resolution of $\sim 60 "$.

From line profiles alone, one cannot distinguish outflows from other bulk motions such as accretion, rotation, infall, shocks, etc. Shepherd and 
Churchwell (1996; hereafter SC96) selected 10 potential outflow sources from the survey of SC95 and made OTF maps in the ${ }^{12} \mathrm{CO}(1-0)$ line and high S/N profiles of ${ }^{13} \mathrm{CO}(1-0)$ using the NRAO $12 \mathrm{~m}$ telescope. The ${ }^{13} \mathrm{CO}$ data were used to correct for optical depth effects in the line wings. Of the 10 sources mapped, 5 have well defined bipolar outflows, 3 had broad line wings but had $\mathrm{S} / \mathrm{N}$ ratios too low to confidently map the line wing emission distributions (due either to bad weather or instrumental problems), and 2 had multiple velocity components in the beam but no evidence for bipolar outflows. For this small sample, we conclude that at least $50 \%$ of the potential outflow sources selected on the basis of broad line wings are indeed produced by outflows. This could be as high as $80 \%$ depending on the status of the 3 sources for which our maps were inadequate to map the line wings.

\section{Outflow Properties}

SC95 and SC96 determined the outflow properties for 7 massive star formation regions using ${ }^{13} \mathrm{CO}$ to correct for optical depth effects in the ${ }^{12} \mathrm{CO}$ line wings. The outflow properties were derived assuming an inclination angle of $45^{\circ}$ to the line of sight. In Table 1 below, the average values of a few of the outflow parameters are compared. Average values for 11 outflows driven by stars with $\mathrm{L}_{b o l}<750 \mathrm{~L}_{\odot}$ were taken from Lada (1985) and those for $\mathrm{L}_{b o l}>10^{3} \mathrm{~L}_{\odot}$ were taken from SC95 and SC96, which includes only B-stars. Values with a superscript $*$ do not include upper limits in the average. As we will see below, one expects the average parameters for outflows driven by more luminous O-stars to be substantially larger than those listed in Table 1.

TABLE 1. Mean Outflow Properties: Low- and High-Mass Stars

\begin{tabular}{lcc}
\hline Parameter & $\begin{array}{c}\text { Mean } \\
\mathrm{L}_{b o l}>10^{3} \mathrm{~L}_{\odot}\end{array}$ & $\begin{array}{c}\text { Mean } \\
\mathrm{L}_{\text {bol }}<750 \mathrm{~L}_{\odot}\end{array}$ \\
\hline $\mathrm{R}_{f}(\mathrm{pc})$ & 0.5 & 0.5 \\
$\mathrm{M}_{f}\left(\mathrm{M}_{\odot}\right)$ & $44^{*}$ & $1.8^{*}$ \\
$\mathrm{~F}_{f}\left(10^{-3} \mathrm{M}_{\odot} \mathrm{km} \mathrm{s}^{-1} \mathrm{yr}^{-1}\right)$ & $8.3^{*}$ & $0.8^{*}$ \\
$\mathrm{~L}_{f}\left(\mathrm{~L}_{\odot}\right)$ & $8^{*}$ & $1.1^{*}$ \\
\hline
\end{tabular}

Clearly, the outflow mass, force, and mechanical luminosity of luminous stars are an order of magnitude or more greater than those of low-luminosity stars. This is also true for the mass flow rate $\dot{M}_{f}$ as illustrated in the $\dot{M}_{f}$ 


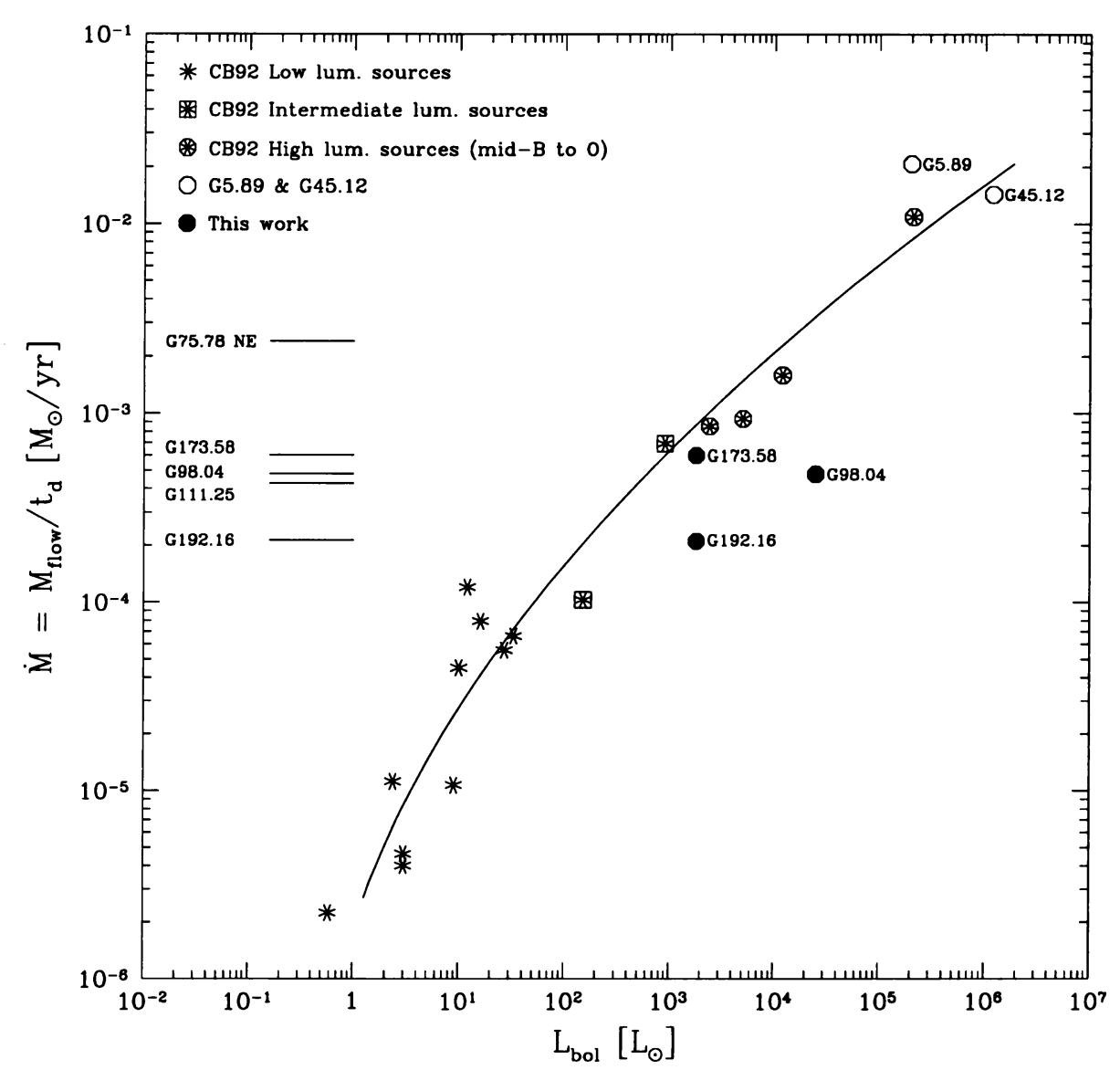

Figure 2. Mass outflow rates versus bolometric luminosity. The solid curve is a second order least squares fit to the data which are indicated by symbols identified in the figure. Figure is from SC96.

versus $\mathrm{L}_{b o l}$ plot shown in Fig. 2 below. Here, in addition to the CB92 sources, those of SC96 and G5.89 and G45.12 from Acord et al. (1997) and Hunter et al. (1997), respectively, are included.

Fig. 2 shows that $\dot{M}_{f}$ is a tight function of $\mathrm{L}_{b o l}$ in the range $1 \leq \mathrm{L}_{b o l} \leq$ $10^{6} \mathrm{~L}_{\odot}$. The solid curve in Fig. 2 is a second order least squares fit to the data which has the form:

$$
\log \left(L_{b o l}\right)=11.2+3.35 \log \left(\dot{M}_{f}\right)+0.24\left(\log \left(\dot{M}_{f}\right)\right)^{2}
$$

with a residual standard error of $\sigma=0.36$. The small dispersion of $\dot{M}_{f}$ 
with $\mathrm{L}_{b o l}$ can be used to infer the bolometric luminosity of the central star as pointed out by SC96 and used by them to argue that the G192.16 and G98.04 outflows are driven by lower luminosity stars than those responsible for the nearby UC HII regions. SC96 also showed that within a given luminosity range, the outflow masses generally increase with age (as expected) and outflows driven by luminous stars are substantially more massive than those of lower luminosity stars of the same age. For example, the Orion A-IRc2 outflow is three orders of magnitude more massive than that of L1448-C which is an order of magnitude older than the Orion outflow. This is a consequence of the much greater mass flux of O-stars relative to lower luminosity stars.

\section{Interferometric Observations}

The study of massive star formation regions suffers from three important disadvantages: none are closer than $450 \mathrm{pc}$, they generally occur in clusters with many lower mass stars close by, and they are deeply embedded in their natal molecular clouds and therefore suffer large visual extinctions. As a consequence, radio (or FIR) interferometric observations are required to determine morphologies on scale sizes $<0.1 \mathrm{pc}$ and to identify the source of the outflow. The observations of G75.78NE by Shepherd, Churchwell, and Willner (1997; hereafter SCW97) and G45.12 by Hunter, Phillips, and Menten (1997; hereafter HPM97) are instructive. Single dish observations indicate a single massive outflow toward G75.78NE which is offset from the UC HII region and apparently is not driven by the ionizing star of the HII region (SC96). BIMA observations of the region with a resolution of $\sim 5$ " by SCW97 reveals at least 3 separate massive outflows in the single dish beam. One of them is centered on a millimeter continuum source, but the other two have no detected radio continuum or IR emission that could be construed as the origin of the outflows. Similarly, single dish observations of G45.12 by HPM97 show a massive outflow centered on the UC HII region. OVRO observations with a resolution of $\sim 2.5$ " reveals at least 2 very massive outflows (see Table 2) only one of which is centered on a compact radio continuum source. Some of the properties of the individual outflows resolved toward G75.78 NE and G45.12 are given in Table 2 below.

Why do we not see a radio continuum source toward all massive outflows if they are driven by massive stars, as claimed? A possible explanation is that during the phase of rapid accretion, matter falls so rapidly onto the YSO that its Strömgren radius is basically coincident with the stellar photosphere and therefore not detectable as a UC HII region during this phase. The FIR emission is probably dominated by the nearby UC HII region and poor spatial resolution prevents one from recognizing multiple sources in 
TABLE 2. Molecular Outflows Associated with OB Stars

\begin{tabular}{lccccccc}
\hline Source & Sp. Type & $\begin{array}{c}\mathrm{M}_{*} \\
\mathrm{M}_{\odot}\end{array}$ & $\begin{array}{c}\log \left(\dot{M}_{f}\right) \\
\mathrm{M}_{\odot} y r^{-1}\end{array}$ & $\begin{array}{c}\mathrm{M}_{f} \\
\mathrm{M}_{\odot}\end{array}$ & $\begin{array}{c}\tau_{d} \\
10^{4} \mathrm{yr}\end{array}$ & Notes & Refs. \\
\hline G5.89 & O6.5 & 28 & -1.59 & 77 & 0.3 & 1,2 & 1 \\
G45.12+0.13 & & & & & & & \\
& O5.5 & 35 & -1.62 & $\sim 4800$ & $\sim 20$ & $1,2,3$ & 2 \\
G45.12+0.13W & & & & & & & \\
G75.78NE-C & B0 & 16 & -2.80 & 58 & 3.7 & 2 & 3 \\
G75.78NE-N & & & -3.45 & 19 & 5.3 & 2 & 3 \\
G75.78NE-E & & & -3.36 & 20.7 & 4.7 & 2 & 3 \\
G98.04+1.45 & O6.5 & 28 & -3.36 & 40 & 9.1 & 1 & 4 \\
G111.25-0.77 & B0.5 & 13 & -3.36 & 16 & 3.7 & 1 & 4 \\
G173.58+2.45 & B2.5 & 7 & -3.22 & 32 & 5.3 & 1 & 4 \\
G192.16-3.82 & B2.5 & 7 & -3.67 & 58 & 27 & 1 & 4 \\
W75N & B1-B2 & 11,8 & -2.92 & 48 & 4.4 & 3 & 5 \\
MonR2(IRS1) & B0 & 16 & -2.95 & 170 & 15 & 1,3 & 7 \\
DR21 & O4-O5 & $>40$ & -1.2 & $\sim 3000$ & 5 & 3 & 6 \\
\hline
\end{tabular}

Notes

1. Single dish results

2. Interferometer results

3. Multiple sources present
References
1. Acord et al. 1997
5. Hunter et al. 1994
6. Garden et al. 1991
7. Tafalla et al. 1997
2. HPM97
3. SCW97
4. SC96
6. Garden et al. 1991
7. Tafalla et al. 1997

the beam. High resolution NIR and intermediate IR observations might be able to resolve this issue. At any rate, high resolution $\mathrm{CO}$ images clearly illustrate that massive star formation regions have multiple, physically close outflows most of which are not driven by the UC HII region in the field.

\section{Origin of the Mass in Massive Outflows}

Outflows driven by luminous stars are often, if not always, more massive than that of the star responsible for driving the outflows. In Table 2, taken from Churchwell (1997), the properties of several massive outflows are tabulated. Churchwell (1997) lists 4 outflows toward G45.12, however, after further analysis HPM97 finds only two outflows. Table 2 has been modified to account for this change; the entries for G45.12 are single dish results which are integrated parameters for all outflows in this region.

What is the origin of the mass in massive star outflows? Four possibilities are: 1) accumulated stellar winds; 2) entrained ISM in stellar bipolar jets; 3) swept-up ISM; and 4) infalling matter diverted into bipolar outflows. I will consider each possibility in the following. 


\subsection{ACCUMULATED STELLAR WINDS}

The winds from $\mathrm{O}$ and $\mathrm{B}$ stars are known to be radiation driven (see reviews and references in Abbott 1985; Lamers and Groenewegen 1990; Pauldrach et al. 1990; and Castor 1993). For stellar winds to supply an outflow of 50 $\mathrm{M}_{\odot}$ over a typical period of $10^{4} \mathrm{yr}$, the average mass loss rate would have to be $\sim 5 \times 10^{-3} \mathrm{M}_{\odot} \mathrm{yr}^{-1}$. This is about two orders of magnitude greater than the highest mass loss rate observed toward O-stars and WR stars. A mass loss rate of this magnitude, if it is radiation driven, cannot be greater than $\frac{L_{*} / c}{v_{\infty}}$ where $L_{*}$ is the luminosity of the star, c is the speed of light, and $v_{\infty}$ is the wind terminal velocity. This requires that the wind terminal velocities must be in the range 0.4 to $4 \mathrm{~km} \mathrm{~s}^{-1}$ for $L_{*}=10^{5-6} \mathrm{~L}_{\odot}$. Such velocities are several times lower than observed molecular outflow velocities. The mass accumulation could be greater if the dynamical lifetimes under-estimate the actual ages of the outflows due to confinement by ambient pressure and the mass loss rates could be greater if multiple scattering is important. In a bipolar outflow, multiple scattering is unlikely to be important because of scattering losses perpendicular to the flow axis. Also, a consideration of the momentum and kinetic energies involved in massive star outflows leads one to conclude that confinement by the ambient interstellar medium is unlikely to increase actual lifetimes by more than a factor of 2 or so beyond that implied by dynamical ages. Thus, neither of these effects appear capable of increasing the accumulated outflows by large enough factors to account for the observed values. It is, therefore, highly unlikely that the outflows are driven by stellar winds which are flowing slower than the outflows themselves. Thus, it also follows that the outflow masses are unlikely to be the result of accumulated stellar winds.

\subsection{ENTRAINED ISM IN STELLAR BIPOLAR JETS}

Let us now consider entrained interstellar matter (ISM) in stellar bipolar jets. The issue here is how much ISM can be entrained in a bipolar jet. The mass entrainment rate per unit area is:

$$
R_{e}=\epsilon \rho_{0} c_{0}
$$

(Cantó and Raga 1991) where $\epsilon$ is the entrainment efficiency $(<<1), \rho_{0}$ is the ambient ISM density, and $c_{0}$ is the speed of sound in the ambient ISM. For a temperature $\mathrm{T}=20-50 \mathrm{~K}$ and density $\mathrm{n}_{\mathrm{H}_{2}} \approx 10^{5} \mathrm{~cm}^{-3}$,

$$
R_{e}\left(g m s^{-1} \mathrm{~cm}^{-2}\right)=\epsilon(0.9-1.4) \times 10^{-14},
$$

If we take the central flow in G75.78NE as an example (see Table 2), we have a flow radius of $0.69 \mathrm{pc}$ and an age of $3.7 \times 10^{4} \mathrm{yr}$. To estimate 
its entrainment area, an approximate cone shape is assumed. For a flow Mach number in the range 10-20, the jet opening angle is expected to be $\theta=2 / \sigma \approx 0.04 \mathrm{rad}=2.3^{\circ}$ from experimental results for a "mixing layer limited" entrainment regime (see Fig. 2 in Cantó and Raga 1991). This gives a total surface area (both lobes) of about $5.8 \times 10^{35} \mathrm{~cm}^{2}$ and an entrained mass:

$$
M_{f}=R_{e} A_{f} \tau_{d} \approx \epsilon(3-5) M_{\odot}
$$

where $\tau_{d}$ is the dynamical lifetime of the outflow and $\mathrm{A}_{f}$ is the entrainment area of the jets. In the case of stellar jets with temperature $\mathrm{T}_{j}=10^{4} \mathrm{~K}$ and an ambient temperature $\mathrm{T}_{0}<100 \mathrm{~K}$, Cantó and Raga (1991) find that the "mixing layer limited" regime is appropriate and that the entrainment efficiency in this regime is $\epsilon<c_{0} / 2 c_{j}$ where $c_{0}$ and $c_{j}$ are the sound speed in the ambient medium and the jet, respectively. For the above conditions, and $\epsilon<0.1$, the total mass of entrained ISM would be $\mathrm{M}_{f}<1 \mathrm{M}_{\odot}$. One could increase the entrained mass by assuming that the stellar mass loss occurs not as a jet but as a flow of matter with much larger opening angles. For example, if $\theta=30^{\circ}$ (effectively increasing the mixing layer area by more than an order of magnitude), $\mathrm{M}_{f}=\epsilon(52-81) M_{\odot}<8 M_{\odot}$. Thus, even in the large opening angle case, one can only entrain a few solar masses in outflows; certainly nothing like the several tens of solar masses observed. Since entrained mass is proportional to the third power of the outflow lobe separation, an under-estimate of this parameter would under-estimate the entrained mass. Failure to correct for inclination of the flow axis to the line of sight under-estimates the lobe separation on average by a factor of $\sqrt{2}$ which results in an average under-estimate of the entrained mass by a factor of $\sim 0.35$, not enough to account for the observed masses. It, therefore, appears that neither accumulated stellar winds nor entrained ISM in stellar jets are capable of providing the mass observed in the outflows from young $\mathrm{OB}$ stars.

\subsection{SWEPT-UP ISM}

It is also possible to set a large mass of ISM into motion along an outflow axis if the density of ISM in the immediate neighborhood of a forming star is large, and if the opening angle of the outflow is large (i.e. not a jet). In this case, the leading boundary of the outflows could act like pistons sweeping up ISM in front of the expanding outflow lobes rather than punching a hole through the ambient medium and sweeping aside the matter as would be the case for a narrow-opening angle jet. There is ample evidence that the densities of ISM is large in the neighborhood of forming massive stars (see Cesaroni et al. 1991; Churchwell, Walmsley, and Wood 1992; Cesaroni, Walmsley, and Churchwell 1992; Hofner et al. 1995; and others). Thus, the 
main issue here is whether the opening angles of massive star outflows are narrow and jet-like or wide. In fact, there is evidence for both.

The Orion A-IRc2 outflow appears to have a fairly wide opening angle as traced by the shock-excited $\mathrm{H}_{2}$ emission obtained by Allen and Burton (1993). The interpretation of the origin and nature of the outflow is controversial, but whatever the correct interpretation, it is clear that the opening angle of the flow is broad on the scale of the $\mathrm{H}_{2}$ emission.

In contrast, the outflows in G45.12, which are among the most massive outflows known, are well collimated. The greater distance of this source, however, prevents us from viewing it with the same resolution possible for Orion. Nonetheless, the high degree of collimation of these very massive outflows produce small cross-sections for interaction with the ISM and are unlikely to sweep-up significant amounts of mass relative to that in the flows.

At this juncture, perhaps all one can say is that swept-up ISM is a possible origin for outflow masses. High resolution images will be required to resolve the issue of whether wide opening angles are common among massive outflows and if they continue to spread with distance from the origin.

\subsection{ACCRETION DRIVEN OUTFLOWS}

A fundamental problem associated with star formation is how they shed angular momentum in order for infalling matter to actually reach the star. The discovery of optical jets and radio bipolar molecular outflows associated with YSOs suggests that this is how they shed angular momentum and permit stars to form. However, the process by which accreting matter is diverted into outflows is not understood, especially for very massive stars. Several models which rely on magnetic fields and rotation have been proposed in the literature (Shu 1991; Pudritz 1988; Shu et al. 1994; Shu 1995; and others at this symposium) for low-mass stars. It is not clear whether any of these are applicable to massive stars. Although the mechanism itself is not understood, it appears likely that deflection of infalling matter into bipolar outflows may be a primary mechanism that feeds outflows of massive YSOs. Some implications of this are examined in the following.

First, it implies that massive YSOs must go through a very rapid accretion phase with a mass accretion rate:

$$
\dot{M}_{a c c}>\dot{M}_{f} \approx 10^{-2}-10^{-3} M_{\odot} y r^{-1} .
$$

$\dot{M}_{a c c}$ must be larger than $\dot{M}_{f}$ since some of the accreting matter ultimately ends up on the central star. $\dot{M}_{a c c}$ also places restrictions on the density 
structure of the accreting matter. Since the infall rate cannot exceed the free-fall rate, if gravity is the only inward force, we have:

$$
\rho(r) \geq \frac{\dot{M}_{a c c}}{4 \pi r^{3 / 2}\left(2 G M_{*}\right)^{1 / 2}},
$$

where $\mathrm{r}$ is the distance from the central star and $M_{*}$ is the mass of the central YSO. For example, for $\dot{M}_{a c c}=10^{-2} M_{\odot} y r^{-1}$ toward an O5 ZAMS star $\left(M_{*} \approx 40 \mathrm{M}_{\odot} ;\right.$ Straizys and Kuriliene 1981), the density of infalling matter at $1000 \mathrm{AU}$ would be $\geq 10^{8} \mathrm{~cm}^{-3}$ and $\geq 10^{5} \mathrm{~cm}^{-3}$ at $\sim 0.5 \mathrm{pc}$. This requirement on the density of infalling matter would provide a natural explanation for the very high densities detected in the immediate vicinity of very compact millimeter continuum sources such as G9.62E and F (Hofner et al. 1995) and CS cores with FIR colors consistent with UC HII regions (Bronfman, Nyman, and May 1996).

Second, if the outflow has achieved its maximum mass (i.e. old and near the end of the bipolar outflow phase), then the outflow mass plus stellar mass gives the minimum mass that a molecular cloud core must have to form a star of a given mass. For example, the central outflow in the G75.78NE region is apparently driven by an early B-star (about 16 $\mathrm{M}_{\odot}$ ) and has an outflow mass of about $58 \mathrm{M}_{\odot}$. Thus, the fraction of the infalling matter actually incorporated into the star is only $\sim 20 \%$ and $\sim 80 \%$ is diverted back outward into the outflow. In this sense, the efficiency of getting matter onto the central star is only about $20 \%$ and one concludes that a cloud core with a mass $\geq 5$ times that of the final star mass is required to form the star. If massive star formation is accompanied by an initial mass function distribution in which most of the mass is in lower mass stars, as appears to be the situation from deep NIR imaging of massive star formation regions (see McCaughrean 1993; McCaughrean and Stauffer 1994; Aspin and Walther 1990, and others), then the mass of the natal molecular cloud core would have to be much greater than the most massive star formed in the association. This may be partly the reason why massive stars are so rare; typical cloud cores generally do not achieve such large masses.

Third, some massive outflows such as NGC6334I (Bachiller and Cernicharo 1990) have flow velocities as high as $100 \mathrm{~km} \mathrm{~s}^{-1}$ or greater. From escape velocity considerations, one can show that these flows must originate from deep in the gravitational well of the central stellar object $(\leq 7$ to $\leq 180 \mathrm{AU}$ for a $40 \mathrm{M}_{\odot}$ central star and a flow velocity in the range 100 to $20 \mathrm{~km} \mathrm{~s}^{-1}$, respectively). The inequality arises because $\mathrm{v}_{\text {flow }} \geq \mathrm{v}_{\text {escape }}$. Thus, observation of the critical region where outflows originate will require spatial resolutions better than 10 to $100 \mathrm{AU}$. 
Fourth, accretion rates of $10^{-2}$ to $10^{-3} \mathrm{M}_{\odot} \mathrm{yr}^{-1}$ will result in most of the stellar UV radiation being absorbed close to the central star, thus delaying the formation of a detectable UC HII region until after this phase of star formation. For example, a density greater than $10^{8} \mathrm{~cm}^{-3}$ of infalling matter toward an O5 star would result in a Strömgren radius $\leq 100 \mathrm{AU}$. This implies that the massive outflows observed to date are likely to be associated with YSOs that have not yet formed a detectable UC HII region. Those that are associated with a UC HII region, such as G5.89, are probably no longer being driven but are relics of an earlier rapid accretion phase. This has the further implication that detection of massive YSOs in their rapid accretion phase or earlier can only be achieved either via their molecular outflows or their thermal dust emission at FIR to mm wavelengths or high excitation molecular line probes, but not from radio free-free emisssion. This does not rule out hot $\left(>10^{5} \mathrm{~K}\right)$, shocked, ionized gas produced by infall onto an accretion disk or in the outflow which might be detectable in very high resolution radio continuum observations.

\section{Summary}

About $90 \%$ of all massive star formation regions have associated high velocity gas. In at least $50 \%$ of these, the high velocity gas is due to bipolar outflows. Molecular outflow properties are tightly correlated with stellar luminosity for $\mathrm{L}_{b o l}$ in the range $\sim 1-10^{6} \mathrm{~L}_{\odot}$. The correlation of $\dot{M}_{f}$ with $\mathrm{L}_{b o l}$ is tight enough that one can predict with reasonable accuracy the stellar $\mathrm{L}_{b o l}$ from a measurement of the outflow rate. The energetic properties of outflows associated with massive YSOs are systematically larger than those of low-mass stars. One also finds that massive star formation regions when observed with high spatial resolution tend to have multiple outflows. In most cases the origin of the outflows are not the UC HII region in the field. The origin of the mass in massive outflows is not likely to be due to accumulated stellar winds or to entrainment of ambient ISM. It could possibly be due to swept-up ISM if the outflow opening angles are large and the flows continue to spread with distance from the origin. It is argued that highly collimated massive outflows are likely to be fed by accreting matter that has been diverted into bipolar outflows. Interesting consequences of this idea are: 1) that massive YSOs must go through a very rapid mass accretion phase with the density structure of the infalling matter dependent on distance from the YSO as $\left.\rho(r) \propto \mathrm{r}^{-3 / 2} ; 2\right)$ the efficiency of getting infalling matter onto a central star is only $\sim 20 \%$ with the rest of the matter being diverted back out into bipolar outflows; 3) the high speed outflows must originate within 10-100 AU of the central YSO; and, 4) during the rapid accretion phase, radio free-free emission is unlikely to be detected. 
Acknowledgements: I would like to thank Deborah Shepherd for her invaluable help in preparing the figures for this review and for many helpful discussions of this subject. I thank Joe Cassinelli for several insightful discussions on stellar winds and stellar mass accretion.

\section{References}

Abbott, D. C. 1985, in Radio Stars, eds. R. M. Hjellming and D. M. Gibson, Reidel, Dordrecht, p. 61

Acord, J. M., Walmsley, C. M., Churchwell, E. 1997, Ap. J., in press

Allen, D. A., Burton, M. G. 1993, Nature, 363, 54

Aspin, C., Walther, D. M. 1990, A\&A, 235, 387

Bachiller, R., Cernicharo, J. 1990, A\&A, 239, 276

Bronfman, I., Nyman, L.-Ä., May, J. 1996, A\&A Suppl., 115, 81

Cabrit, S., Bertout, C. 1992, A\&A, 261, 274 (CB92)

Cantó, J., Raga, A. C. 1991, Ap. J., 372, 646

Castor, J. I. 1993, in Massive Stars: Their Lives in the Interstellar Medium, eds. J. P. Cassinelli and E. B. Churchwell, A. S. P. Conf. Series, 35, 297

Cesaroni, R., Walmsley, C. M., Churchwell, E. 1992, A\&A, 256, 618

Cesaroni, R., Walmsley, C. M., Kömpe, C., Churchwell, E. 1991, A\&A, 252, 278

Churchwell, E. 1997, Ap. J. Lett., in press

Churchwell, E., Walmsley, C. M., Wood, D. O. S. 1992, A\&A, 253, 541

Edwards, S. Ray, T, Mundt, R. 1993, in Protostars and Planets III, eds. E. H. Levy and J. L. Lunine, Univ. Arizona Press, Tucson\&London, p. 567

Fukui, Y., Iwata, T., Mizuno, A. Bally, J., Lane, A. P. 1993, in Protostars and Planets III, eds. E. H. Levy and J. L. Lunine, Univ. Arizona Press, Tucson\&London, p. 603

Garden, R. P., Hayashi, M., Gatley, I., Hasegawa, T., Kaifu, N. 1991, Ap. J., 374, 540

Hofner, P., Kurtz, S., Churchwell, E., Walmsley, C. M., Cesaroni, R. 1995, Ap. J., 460, 359

Hunter, T. R., Phillips, T. G., Menten, K. M. 1997, Ap. J., in press (HPM97)

Hunter, T. R., Taylor, G. B., Felli, M., Tofani, G. 1994, A\&A, 284, 215

Lada, C. J. 1985, Ann. Rev. A\&A, 23, 267

Lamers, H. G. J. L. M. \& Groenewegen, M. A. T. 1990, in Properties of Hot Luminous Stars, ed. C. D. Garmany, A. S. P. Conf. Series, 7, 189

McCaughrean, M. 1993, in Massive Stars: Their Lives in the Interstellar Medium, eds. J. P. Cassinelli and E. B. Churchwell, A. S. P. Conf. Series, 35, 80

McCaughrean, M., Stauffer, J. R. 1994, A. J., 108, 1382

Pauldrach, A. W. A., Puls, J., Gabler, R., Gabler, A. 1990, in Properties of Hot Luminous Stars, ed. C. D. Garmany, A. S. P. Conf. Series, 7, 171

Pudritz, R. E. 1988, in Galactic and Extragalactic Star Formation, eds. R. E. Pudritz and M. Fich, Kluwer Acad. Pub., Netherlands, p. 135

Shepherd, D. S., Churchwell, E., Willner, D. J. 1997, Ap. J., in press (SCW97)

Shepherd, D. S., Churchwell, E. 1996, Ap. J., 472, 225 (SC96)

Shepherd, D. S., Churchwell, E. 1995, Ap. J., 457, 267 (SC95)

Shu, F. H. 1991, in The Physics of Star Formation and Early Evolution, eds. C. J. Lada and N. D. Kylafis, Kluwer Acad. Pub., Dordrecht, Netherlands, p. 365

Shu, F. 1995, Rev. Mex. A. A. (Serie de Conf), 1, 375

Shu, F., Najita, J., Ostricker, E., Wilkin, F., Ruden, S., Lizano, S. 1994, Ap. J., 429, 781

Straizys, V., Kuriliene, G. 1981, Ast.\&Sp. Sci., 80, 353

Tafalla, M., Bachiller, R., Wright, M. C. H., Welch, W. J. 1997, Ap. J., 474, 329 